Article

\title{
Toward a New Science of Information
}

\section{Wolfgang Hofkirchner}

Institute of Design and Technology Assessment, Vienna University of Technology, Austria; Home Office: Steinbrechergasse 15, 1220 Wien, Austria; E-Mail: wolfgang.hofkirchner@tuwien.ac.at

Received: 4 May 2011; in revised form: 8 June 2011 / Accepted: 9 June 2011 /

Published: 16 June 2011

\begin{abstract}
Currently, a Science of Information does not exist. What we have is Information Science that grew out of Library and Documentation Science with the help of Computer Science. The basic understanding of information in Information Science is the Shannon type of "information" at which numerous criticisms have been levelled so far. The task of an as-yet-to-be-developed Science of Information would be to study the feasibility of, and to advance, approaches toward a more general Theory of Information and toward a common concept of information. What scientific requirements need to be met when trying to develop a Science of Information? What are the aims of a Science of Information? What is the scope of a Science of Information? What tools should a Science of Information make use of? The present paper responds to these questions.
\end{abstract}

Keywords: science of information; information science; information studies

\section{Science of Information-An Idea Whose Time Has Come}

Currently, a Science of Information does not exist. What we have is Information Science. Information Science is commonly known as a field that grew out of Library and Documentation Science with the help of Computer Science: it deals with problems in the context of the so-called storage and retrieval of information in social organizations using different media, and it might run under the label of Informatics as well. A Science of Information, however, would be a discipline dealing with information processes in natural, social and technological systems and thus have a broader scope. This is how the term Information Science is understood by a community of academics from different fields of science, engineering, humanities and arts who have been gathering around a 
conference series, a mailing list and a website with the abbreviation "FIS" (Foundations of Information Science) for more than a decade.

Currently, no (Unified) Theory of Information is available. Information Theory exists: it is a branch of mathematics and engineering science inaugurated by Claude E. Shannon's paper "A Mathematical Theory of Communication” [1]. That contribution dealt with the problem of keeping the signal-noiseratio in communication transmission channels (hence the designation "channel" model) under control-a war-related problem. The entity that Shannon's formalism intended to measure soon came to signify "information”. A (Unified) Theory of Information would have to deliberate about whether to generalize a framework that specifically focuses on syntactic aspects and omits semantic ones, yet reaches out to fields that require reference to semantics. This has been precisely the brunt of many criticisms ever since. The result has been alternative theoretical approaches, none of which ever succeeded in being recognized by the overall scientific community as a more general Theory of Information that would, in fact, incorporate Shannonian Information Theory.

Numerous criticisms have been levelled at the Shannon type of "information". One type of criticism has grown out of deliberations of single disciplines and points to particular problems. These problems mainly involve issues of how meaning enters the stage of information (a semantic question) and of how systems respond to information input (a pragmatic question).

Besides the attempts to complement the Shannon information concept from a particular point of view, there has been a search for a concept that can overcome the shortcomings by integrating the various aspects of information processes. The useful aspects, if any, of the Shannonian term should be included as a special case when extending the restricted information theory into a new, universal theory. Clearly, transdisciplinary undertakings that strive for the bigger picture tend to be affiliated to philosophy and cross-disciplines such as cybernetics, system theory, evolutionary theory.

All these transdisciplinary initiatives make an even stronger case for substantiating the apparently unsubstantiated use of the term "information" throughout sciences than earlier point-by-point criticisms. Combined, they form constituents of a huge melting pot of scientific exchange and dispute. They have helped heat up the debate to a point where a critical value seems to have been reached. This is equivalent to a chaos point: either the debate will cool down and things will remain as they were, or a leap in quality will emerge from the chaos and bring about change and new order.

Hence, a Science of Information is an idea whose time has come!

\section{Aims, Scope and Tools of a Science of Information}

The chaos point we have reached provides a window of opportunity for a paradigm shift. The task of an as-yet-to-be-developed Science of Information would be to study the feasibility of, and to advance, approaches toward a more general Theory of Information and toward a common concept of information while constantly being aware of a potential failure of the project.

What scientific requirements need to be met when trying to develop a Science of Information? In other words, what is, in scientific terms, required by a future Science of Information (see Doucette et al. [2])? To be ready for the paradigm shift, we must anticipate now what will be required in the future. 
Three contexts — each having specific criteria — can be distinguished in terms of what makes science scientific:

- a context of application in which scientific knowledge is used to solve problems and is transformed into technologies, whether material or ideational;

- a context of justification in which scientific knowledge is critically exposed to possible refutations and corroborated in as far as it is not refuted, and theories are comparatively assessed;

- a context of discovery in which scientific knowledge is conjectured and theoretical assumptions are formulated in relation to empirical findings.

The first context concerns the objective, the end, the aims for which the scientific endeavor is undertaken. It fulfils the task of solving a problem that arises from practice in society and it is practically guided by the aims.

The second context refers to the object of the inquiry; this is the starting point that determines the domain, the scope to be researched.

The third context is about objectivation, i.e., methods that help objectivate or, substantiate, the findings; these strategies are the means to transport the scientific enterprise from the starting point to its end. This context is the tools used.

Accordingly, each science can be described in terms of aims, scope, and tools.

A key assumption is that some relationship exists that connects aims, scope and tools: they build a three-levelled architecture with aims on top, scope at an intermediate position and tools at the bottom. Thus, despite a relative autonomy of each of the levels, aims define the possible scope (particular interests demand a particular section of reality to be selected for investigation), and the scope defines possible tools (not every method is appropriate to study a particular object). In reverse, specific tools determine the variety of the scope that is realized (the constitution of the object), and a particular scope determines the variety of possible aims to be served (the findings cannot be used for any practical guidance).

At each level, criteria apply that make scientific thinking distinct from (yet merely a thoroughly reflected continuation of) common sense:

- In the aftermath of the Positivism debate in German sociology between the Frankfurt School type Critical Theory and positivist Critical Rationalism, philosophy of science considerations seem to have agreed that value-free science does not exist. Clearly, technology serves humane values. Technology assessment must take into account the interests of those affected by technology and examine whether or not their needs, as well as general interests, are met. The dispute is mostly about which values to prioritize. Such a dispute requires explicitly formulating the values implicit in scientific and technological developments.

- Findings shall be anchored in theories which, preferably as universal implications, are statements describing general and necessary properties or general and necessary relations that cover the object of inquiry as a whole. As a rule, if a theory is challenged by a counterexample not obeying the law, another theory is sought to explain this phenomenon as well as all phenomena the old theory could explain. In times of normal science-to use the terminology Thomas S. Kuhn [3] coined-no such need arises. Other times call for a scientific revolution (whose reach may differ according to the issues at hand) and for paradigms that compete with 
each other (which may range from different schools of thought within a subdiscipline to entirely contrasting world views).

- Logically, no induction compellingly leads from concrete empirical grounds to theoretical generalizations. Nonetheless, many methods have been devised by different disciplines to support the creative construction of theories. The scientific debate revolves around immanent and transcendent criticisms of whether or not the methods applied are appropriate.

What are the aims of a Science of Information?

What is the scope of a Science of Information?

What tools should a Science of Information make use of?

The proposal here is to determine the three levels of a desired Science of Information ex negativo, that is, in comparison or contradistinction to the features characterizing the state-of-the-art of normal science that conducts information research. This is because they do not meet the needs of societal development today.

\section{1. “Normal Science” Information Studies}

On each science level, the current paradigm in information studies faces a pluralism that extends to a cleft between divergent tendencies. It is a cleft

1. between technocratic and ivory tower perspectives on information at the aims level,

2. between reifying and deconstructive perspectives on information at the scope level, and,

3. between reductionistic and projectivistic as well as disjunctivistic perspectives on information at the tools level.

\subsubsection{Technocracy vs. Ivory Tower}

There is a traditional distinction between applied sciences and basic sciences which-in a widely shared belief-is becoming increasingly blurred. The image of an engineer employed in a private lab and taking orders from his/her employer versus an academic merely satisfying his/her curiosity is old-fashioned and outdated. It is true that scientists enjoy a certain freedom of research within given financial, policy and other constraints. This, however, reflects the fact that research and development, starting in the late 1970s, have been streamlined world-wide according to neoliberal economic policies of liberalization, privatization, and deregulation. It is less a reflection of the general statement that science at any given time is part of society and thus responsive, be it directly or indirectly, to historically developing societal needs. This helps explain why, in developed/rich countries, many disciplines, especially in the humanities, are publicly stigmatized as being beautiful but useless and suffer cuts and total suspensions. Short-sighted economic interests have taken command in scientific affairs. Thus it still makes sense to distinguish between a business-driven development of science and technology and l'art pour l'art activities.

Accordingly, information concepts might either provide some foundation for applications of Information and Communication Technologies or be far detached from real-world problems.

Given the confines of economic profitability and competitiveness, the credo of technocracy is in force. Its credo: "realize everything that is feasible”. This falsely presupposes that everything feasible 
(again, assuming it is economically reasonable) is also desirable. Accordingly, a reflective, theoretical, deliberation of norms, values, morals would be superfluous or, at best, replaced by a posteriori, empirical inquiries about the acceptance of technology by users. This, however, detracts from considering more fundamental problems than those of profitability. The historically most striking example for an information concept in line with this tendency is the one developed in the context of a purely technological problem (arising from military concerns) in the Bell Laboratories after World War II: Shannon’s channel concept.

Conceptualizations of information devoid of considerations about potential far-reaching impacts on society are prone to being subjected to dominating economic, political, military interests. This may ultimately also be true of conceptualizations not intended to serve any specific purpose but kept within the walls of the ivory tower. Although such cases resist subsumption under instrumental reason, they are nonetheless produced under determinate historical circumstances that may unwillingly instil certain inherent values. Anything imaginable may be influenced by the state-of-the-art of already produced imaginations. Complete refusal of applicability is unreasonable.

\subsubsection{Reification vs. Deconstruction}

Objects of theorizing and empirical investigation might be categorized as being a structure or being a process. Both categories can be viewed as being mutually exclusive and, together, exhausting the possible multitude.

Accordingly, information may be viewed as having the quality of a thing or of an event that occurs.

Screening current information concepts along this line reveals two such clusters of common perspectives.

The first cluster is composed of two sub-clusters.

- A first sub-cluster of information concepts/theories consists of those that regard information as a given. This has been termed "potential” information or "structural” information. The structural sciences deal with that topic. They hold that matter is always in a certain shape, gestalt, or form, and this form is information (Bernd-Olaf Küppers is a prominent advocate of this position, which is espoused with the notion of "Strukturwissenschaften"- "Structural Sciences"introduced in the 1970s by Carl Friedrich von Weizsäcker, [4]). This form is something fixed and is equivalent to a thing.

- The second sub-cluster focuses on the transmission aspect. From that angle, information lies not in the structure but in that which is transmitted from a sender to a receiver via a channel (and might then be disturbed by noise). That is the classical view introduced by Shannon and is the forebear of all communication models [1]. The transmission view of information does not see information as an event. Information is that which is carried by the signals through the channel. That which flows or floats here is sometimes referred to as "free" information. This "free" information is also something fixed, a thing that is carried.

Both sub-clusters hypostatize the phenomenon of information, they ontologize it in a specific way, they reify it. 
Cluster number two-based on the sender-receiver-model, but extending and transcending itfocuses on what happens with, and within, the receiver. Information is ultimately not that which is transmitted-pursuant to this perspective-but that which is processed by the receiver or, more precisely, which is produced by the receiver. The receiver, by processes of decoding, attaches a meaning to the message and thereby produces "actual" information. This is the leitmotif of all developments in communication studies, in particular cultural studies, which strive to complement or depart from the channel model. The German sociologist Luhmann deconstructed the notion of information in his social systems theory [5,6]. According to him, information is by no means something lying around in the environment and waiting to be picked up. Accordingly, information cannot be transmitted either. Information is an event that occurs when expectations are frustrated and as a result of that difference another difference is triggered.

This information, being an event, is hard to get a hold on. The Austrian computer pioneer Heinz Zemanek, who was involved in introducing informatics - which he did not want to call "computer science" - at the tertiary education level in Austria, insisted on the social nature of information. This makes it impossible to quantify or measure [7].

Thus, information melts away as something fuzzy, intangible and inconceivable.

\subsubsection{Reductionism vs. Projectivism and Disjunctivism}

Reductionism, projectivism, and disjunctivism are different ways of conceiving the complexity of objects being investigated. They manifest themselves in the methodology of information studies.

Two different levels can be identified: the first is related to the philosophical foundation of the methodology used, the second is related to the disciplinary foundation of the methodology used.

The philosophical considerations underlying the methodology seek the essence of information, the nature of information, the substance which constitutes it. The question of what information is must be answered in relation to the essence, nature, and substance of matter.

- The first answer is that information is a substance equivalent to matter. This substance is conceived of as something material, making information something material. Such an answer is material(istic) monism: everything is like matter and the same holds true for information. This is materialism. Materialism in that sense is reductionistic. Under the premise that information is more complex than matter, information is reduced to matter.

- A second answer states that this substance is immaterial, making information something immaterial. This answer is immaterial (ideal, idealistic, ideational, informational) monism, idealism matter is also like mind (information). Varieties include Platonism and Radical Constructivism. This, under the same premise as before, is projectivistic because information is projected onto matter.

- Another answer is that matter and information do not share the substance: they are essentially different in nature. Matter is material and information is not: this is the answer of dualism. Such an answer, again under the same premise as before, is disjunctivistic; it disjoins information from matter. This gives rise to yet another question: are these two substances inert and non-reactive to each other or do they interact and, if so, how then can one side of the duality affect the other side? How is it possible that matter influences mind (information)? How is it 
possible that mind (information) affects matter? Efforts in this direction include the Cartesian tradition (note that Descartes contended that the interaction between res cogitans and res extensa takes place in the pineal gland) and, more recently, John Eccles, who together with Karl Raimund Popper identified the synapses as the location where mind and brain interact [8].

The branch-specific considerations underlying the methodology relate to the gap between the natural and the engineering sciences (including formal sciences) on the one hand and the arts and humanities (including the social sciences) on the other hand. This dates back to the 17th century and to philosophers such as, again, René Descartes. The gap between the two branches in science reached its peak in the late $19^{\text {th }}$ century with the works of Neo-Kantian philosophers, scientists, and literary intellectuals such as Wilhelm Windelband and Heinrich Rickert. Wilhelm Windelband [9], for example, introduced the disjunction between "nomothetic" (meaning: positing laws) and "ideographic" (meaning: describing events): these would remain, existing alongside one another, as the final, incommensurable forms of our notions about the world. Today this cleft is known as C. P. Snow's dilemma of the two cultures, which he bemoaned in 1959 and 1963 [10]. Although John Brockman, an author from the USA, foresees the emergence of a third culture "founded on the realization of the import of complexity, of evolution" [11], this is by no means mainstream. The cleft is characterized by the preponderance of either the analytical method or phenomenological and hermeneutic methods, of so-called third-person science versus first-person science, of an externalist versus an internalist view: each characterizes one or the other tendency. The same holds true for the information concepts.

- The first approach is, methodologically, reductionistic. It reduces different qualities of the phenomena under investigation to one and the same quality, typically the quality which is the simplest.

- The second approach is characterized by a humanistic rationality which is ignorant of the field of science and technology. Methodologically, there are two possibilities. The first projects a particular quality (typically the most complex one) onto phenomena which lack this quality, and then purports to discover them there. Properties of information in nonhuman domains are usually extrapolated from properties of information in the human domain (anthropo(socio)morphism). The second gives up the attempt at a subsuming, though unifying, solution and argues instead in favor of a lack of comparability of the given phenomena in nature and society. In this dichotomizing, disjunctive view, information is exclusively ascribed to the human domain. Going even further, it may exclusively be ascribed to particular incidences within the human domain.

\subsection{A Science of Information Paradigm}

Accordingly, information studies appear to have already passed the normal science phase and to have entered a critical phase. This is because, at each level—be it aims, scope or tools-a possible position is countered by a possible counter position. Precisely these discrepancies form an obstacle to unification. Progress in the direction of a Science of Information implies efforts to overcome these divides. 
Those efforts should help take up what is important while discarding the one-sidedness of any particular theory in the field. The search for integration potentials between different theories should do justice to their input and at the same time reflect caveats concerning possible blind spots.

\subsubsection{Ensuring Futurability}

Concerning the opposition of applied science and basic science, Pasteur's Quadrant offers a solution. Donald Stokes [12] produced a 4 times 4 matrix with the "Quest for fundamental understanding?" as one dimension and "Consideration of use?" as the second one, with yes and no answers for each. For him, Thomas Alva Edison is the role model for pure applied research, Niels Bohr the model for pure basic research: Louis Pasteur, however, is paradigmatic for a new way of doing science, namely "use-inspired basic research".

A Science of Information certainly belongs to Pasteur's Quadrant and must be open to practical aspects if it is to successfully support the search for a fundamental understanding of information. Success, however, must not be understood in a restricted economic, political, military or technological sense. What is needed is openness in considering the great challenges facing humanity today and prioritizing values accordingly. The raison d'etre of a Science of Information is to provide society with a means of enhancing its problem-solving capacity vis-à-vis these challenges, to provide society with a future, to make it futurable.

The underlying problems consist of frictions in the functioning of society, environment and technology. Problem-solving activities seek information applications that reduce the frictions. Accordingly, a Science of Information would be the safeguard against the loss of control, it would guarantee the stabilization of development and the maintenance of society. In this sense, it is destined to become a, if not the, science for the information society.

The vision of a "good society" must serve as a point of departure [13]. In this sense, a Science of Information is normative. Technological applications are to be closely reviewed, and the question is: are they apt to serve the purpose of a good society?

The design process must start by identifying a societal problem and then proceed with the search for appropriate applications (and not vice versa, as is done under technocratic premises). In that context, a Science of Information implies a transcendence from the scientists to the stakeholders and those affected by the results of research. It implies a transformation into a new science that is human-centered, democratic, participatory, such as Helga Nowotny’s “Mode-2” science [14].

\subsubsection{Catching the Ephemeral}

A future Science of Information is not merely normative. It also does justice to the factual. In evaluating the potential that selects the desired, it also accounts for the potential that is given with the actual. Accordingly, it is clear that the domain of this Science of Information encompasses anything that promotes or stalls a good society. It identifies the frictions that cause malfunctions in society, environment and technology. And it seeks potentials of ephemeralization.

Importantly, society has used the informational revolution to, theoretically, boost its potential to reduce frictions wherever they appear. This reflects the ongoing information processes between all parts of society, environment and technology. What is missing is a science to guide investigations into 
these frictions. Thus each real-world system-whether social, natural, or artificial—is involved in information processes. This calls for theorizing about and dealing with such systems in order to master those information processes that may help downsize the frictions causing the global challenges.

To understand these phenomena, information must be understood as both structure and process, i.e., as a "structuration process" [15] in which processes produce structures that, in turn, structure the processes, that is, function as both constraints and enablers for the continuation of the processes. In fact, frictions in the information processes depend on the very relationship of constraining and enabling.

Information can be viewed as something overarching the whole bandwidth of different and diverse structuration processes in our universe and manifesting itself in a variety of phenomena.

\subsubsection{Taking the Blind Men’s Perspective}

The investigation must comprise the wide range of matter pointed out above. Thus, a Science of Information cannot, with reference to the tools, afford to neglect any potentially fruitful and elucidating methodological approaches. Likewise it must not fail in putting the puzzle of findings together and in synthesizing the manifold analyses. This would help transcend the borders of disciplines and strive for the unity of science based on a unifying approach, without subjecting any thinking to uniformity.

Transdisciplinarity defines concepts that go beyond the meaning of multi- and even interdisciplinarity. Multidisciplinarity means the unrelated coexistence of monodisciplinary accounts, and interdisciplinarity means the casual establishment of relations between monodisciplines without feedback loops that lastingly impact their repertoire of methods and concepts. Transdisciplinarity, in contrast, comes into play when each discipline is engaged in the collaborative undertaking of constructing a common foundation of methods and concepts, of which its own methods and concepts can be understood as a type of instantiation. Transdisciplinarity does not mean abolishing disciplinary knowledge, but grasping for a bigger picture. Methods used in different approaches may cooperate when viewed as methods of subdomains of the new Science of Information-from the science of the information society to the philosophy of science, which are linked via different levels of abstraction including social and human sciences, engineering, natural and formal sciences, and cross-disciplines such as systems theory.

But how can the divide between materialist and idealist monism and idealist dualism, and the divide between the two cultures, be successfully bridged? The answer is like in the story of the elephant and the blind men (or the men in a dark room), each of whom touches a different part of the elephant and mistakes the part for the whole [16]. Accordingly, none of the various existing information concepts/theories should take its perspective as being absolute but, rather, as being complementary to the other perspectives.

This calls for a way of thinking that goes beyond reductionism, projectivism, disjunctivism. What is needed is "unitas multiplex", as the French philosopher and sociologist Edgar Morin calls it [17]: "It means understanding disjunctive, reductive thought by exercising thought that distinguishes and connects. It does not mean giving up knowledge of the parts for knowledge of the whole, or giving up analysis for synthesis, it means conjugating them. This is the challenge of complexity which ineluctably confronts us as our planetary era advances and evolves.” 
This is the integrative way of thinking that a Science of Information will need to incorporate.

\section{Conclusion}

This paper discussed scientific requirements that need to be met when trying to develop a Science of Information. This development signifies a scientific revolution. Taking up the philosophy-of-science differentiation of aims, scope and tools, information studies in the phase of normal science are characterized by three clefts: one cleft between technocratic and ivory tower perspectives, another cleft between reifying and deconstructive perspectives, and a third cleft between reductionistic and projectivistic as well as disjunctivistic perspectives.

A Science of Information implies efforts to overcome these divides. On the aims level, it sets out to be a science for the information society which means that it must be use-inspired, though basic research: It aims to enhance society's problem-solving capacity when confronted with challenges that threaten to terminate civilized life on planet Earth. This normative touch has implications for the scope and tools levels. On the scope level, a Science of Information focuses on those information processes that may help downsize the frictions causing the global challenges: it is a science about the information society that is made up of different kinds of information-generating systems. And on the tools level, a Science of Information is a transdiscipline that integrates different methods to do justice to the interplay of different systems: this integration is carried out by virtue of the information society that instigates complex thinking so as to make a Science of Information a science by virtue of the information society.

Contributions on either level are required to help bring about the paradigm shift to the new Science of Information.

\section{References}

1. Shannon, C.E. A mathematical theory of communication. Bell Syst. Tech. J. 1948, 27, 379-423, 623-656.

2. Doucette, D.; Hofkirchner, W.; Bichler, R.; Raffl, C. Toward a new science of information. Data Sci. J. 2007, 6, S198-S205.

3. Kuhn, T.S. The Structure of Scientific Revolutions; University of Chicago Press: Chicago, IL, USA, 1962.

4. Küppers, B.O. Die Strukturwissenschaften als Bindeglied zwischen Natur- und Geisteswissenschaften. In Die Einheit der Wirklichkeit; Küppers, B.O., Ed.; Wilhelm Fink: München, Germany, 2000; pp. 89-105.

5. Luhmann, N. Soziologische Aufklärung; Westdeutscher Verlag: Opladen, Germany, 1981.

6. Luhmann, N. Soziale Systeme; Suhrkamp: Frankfurt, Germany, 1984.

7. Zemanek, H. Informationsverarbeitung und die Geisteswissenschaften. Anzeiger der phil.-hist. Klasse der Österreichischen Akademie der Wissenschaften 1988,.124, 199-225.

8. Popper, K.R.; Eccles, J.C. The Self and Its Brain; Springer: Berlin, Germany, 1977. 
9. Windelband, W. Geschichte und Naturwissenschaft; Rede zum Antritt des Rektorats der Kaiser-Wilhelms-Universität-Straßburg, gehalten am 1. Mai 1894. Available online: http://www.fh-augsburg.de/ harsch/germanica/Chronologie/19Jh/Windelband/win_rede.html (accessed on 8 March 2008).

10. Snow, C.P. The Two Cultures. A Second Look; Cambridge University Press: Cambridge, UK, 1998.

11. Brockman, J. The Third Culture. Beyond the Scientific Revolution; Simon and Schuster: New York, NY, USA, 1995; pp. 20-21.

12. Stokes, D. Pasteur's Quadrant. Basic Science and Technological Innovation; Brookings Institution: Washington, DC, USA, 1997.

13. Hofkirchner, W. ICTs for a Good Society. In Information and Communication Technologies, Society and Human Beings, Theory and Framework, Honoring Gunilla Bradley; Eriksson, D., Mirijamdotter, A., Eds.; Information Science Reference, Hershey: New York, NY, USA, 2011, pp. 434-443.

14. Gibbons, M.; Nowotny, H. The Potential of Transdisciplinarity. In Transdisciplinarity: Joint Problem Solving among Science, Technology, and Society, an Effective Way for Managing Complexity; Thompson Klein, J., Grossenbacher-Mansuy, W., Häberli, R., Bill, A., Scholz, R.W., Welti, M., Eds.; Birkhäuser: Basel, Switzerland, 2001.

15. Giddens, A. The Constitution of Society, Outline of the Theory of Structuration; Polity Press: Cambridge, UK, 1984.

16. Blind Men and an Elephant. Available online: http://en.wikipedia.org/wiki/Blind_Men_and_an_ Elephant (accessed on 8 March 2008).

17. Morin, E. Seven Complex Lessons in Education for the Future; UNESCO: Paris, France, 1999; p. 19, p. 25.

(C) 2011 by the authors; licensee MDPI, Basel, Switzerland. This article is an open access article distributed under the terms and conditions of the Creative Commons Attribution license (http://creativecommons.org/licenses/by/3.0/). 